\title{
Evaluación de un campo clínico de enfermería del área materno infantil
}

\author{
Dra. Cristina Hortensia Saavedra Vélez \\ cindyenfe@hotmail.com \\ ORCID 0000-0002-9639-8599 \\ Académico de tiempo completo. \\ Universidad Veracruzana. Facultad de Enfermería, \\ Región Xalapa \\ Dra. María Elena Columba Meza Zamora \\ meze53@hotmail.com \\ Académico de tiempo completo. \\ Universidad Veracruzana. Facultad de Enfermería \\ Región Xalapa \\ Dr. Omar Sánchez de la Cruz \\ omsan1969@yahoo.com.mx \\ Profesor de asignatura \\ Universidad Veracruzana. Facultad de Enfermería, \\ Región Xalapa \\ ME Beatriz Adriana García Rivas \\ beatrigarcia28@gmail.com \\ Profesor de asignatura \\ Universidad Veracruzana. Facultad de Enfermería, \\ Región Xalapa
}

\section{RESUMEN}

Introducción: La práctica clínica es fundamental en la formación de enfermería. Incluye en función de objetivos curriculares, servicios acordes, estrategias de enseñanzaaprendizaje congruentes, ambientes de aprendizaje, métodos de supervisión y de evaluación. Los campos clínicos deben ser evaluados por académicos y estudiantes.

Objetivo: Evaluar un campo clínico del área materno infantil. Metodología. Estudio cuantitativo, descriptivo, longitudinal, efectuado de 2015 a 2019 en un Hospital de 2do nivel. Técnica e instrumento: Encuesta y cuestionario que explora: Organización, ambiente laboral y de aprendizaje, relación teoría-práctica, seguimiento y evaluación. Población: Estudiantes que cursaron la experiencia educativa. Muestra: 81 alumnos ubicados en campo clínico seleccionado. Resultados: Campo clínico y horario adecuados $80 \%$ y $100 \%$, no adecuada la duración $25 \%$. 100\% refirió motivadores el entorno, 
condiciones y experiencia. Relaciones interpersonales satisfactorias entre $90 \%$ y $100 \%$. Para $+80 \%$ son correlacionales saberes previos y los requeridos. $-80 \%$ opinó que la clínica capacita para el área materno infantil. $+80 \%$ considera adecuado el sistema de evaluación y supervisión. Conclusión: Los resultados reflejan que el campo clínico es adecuado. Como buena praxis la comunidad académica debe identificar oportunidades para incidir, mediante análisis, reflexión, discusión académica e investigación educativa para mejorar la enseñanza clínica.

Palabras clave: campo clínico; evaluación; enfermería 


\title{
Evaluación de un campo clínico de enfermería del área materno infantil
}

\begin{abstract}
Introduction: Clinical practice is essential in nursing training. It includes, based on curricular objectives, consistent services, consistent teaching-learning strategies, learning environments, monitoring and evaluation methods. Clinical fields must be evaluated by academics and students. Objective: To evaluate a clinical field of the maternal and child area. Methodology. Quantitative, descriptive, longitudinal study, carried out from 2015 to 2019 in a 2nd level Hospital. Technique and instrument: Survey and questionnaire that explores: Organization, work and learning environment, theory-practice relationship, monitoring and evaluation. Population: Students who completed the educational experience. Sample: 81 students located in selected clinical field. Results: $80 \%$ and $100 \%$ adequate clinical field and time, not adequate duration $25 \% .100 \%$ referred motivators to the environment, conditions and experience. Satisfactory interpersonal relationships between $90 \%$ and $100 \%$. For $+80 \%$, previous and required knowledge are correlational. $-80 \%$ thought that the clinic provides training for the maternal and child area. $+80 \%$ consider the evaluation and supervision system adequate. Conclusion: The results reflect that the clinical field is adequate. As good practice, the academic community must identify opportunities to influence, through analysis, reflection, academic discussion and educational research to improve clinical teaching.
\end{abstract}

Keywords: clinical field; evaluation; nursing

Artículo recibido: 18. Julio. 2021 Aceptado para publicación: 08. Agosto. 2021 Correspondencia: cindyenfe@ hotmail.com Conflictos de Interés: Ninguna que declarar

\section{INTRODUCCIÓN}


De acuerdo a la Secretaría de Salud (2012) el término Campo clínico de enfermería hace referencia al "Establecimiento para la atención de la salud del Sistema Nacional de Salud o bien alguna de sus áreas o servicios que cuentan con la infraestructura, equipamiento, población usuaria, personal médico, de enfermería, paramédico y administrativo, que está autorizado para que se apliquen los conocimientos derivados de los programas académicos del plan de estudios de enfermería y alcanzar los objetivos curriculares.” Para Maroto (2017) un escenario clínico de aprendizaje es el espacio considerado como el "aula clínica donde cada discente se enfrenta a experiencias de aprendizaje que involucran el ejercicio supervisado de la profesión que se estudia" y que en el caso de carreras de ciencias de la salud por tanto corresponde a hospitales, clínicas de servicio, hospitales escuela, etc, que favorecen la adquisición y desarrollo de habilidades, destrezas y actitudes propias de cada disciplina.

Durante (2012, citado por Maroto 2017) relaciona esta experiencia de enseñanza con el aprendizaje que se adquiere en ambientes de trabajo propios de la profesión, al atender a usuarios sanos o enfermos que demandan atención específica de enfermería en materia de salud.

La selección de campos clínicos, por parte de las entidades educativas, se fundamenta en la idoneidad de estos, en concordancia con los objetivos curriculares. Para el caso que nos ocupa la práctica del área materno infantil se lleva a cabo en unidades hospitalarias de 2 do nivel que cuentan con servicios que brindan atención a mujeres durante el embarazo, parto y puerperio, así como al niño desde recién nacido hasta adolescencia.

Toda vez que la práctica en escenarios clínicos, es un aspecto central en la formación profesional de los alumnos de enfermería, es ahí donde se enfrentan a experiencias de aprendizaje en contextos institucionales con sus correspondientes características de infraestructura, nivel de atención, equipamiento, recursos humanos y normativa.

$\mathrm{Al}$ desarrollarse en ambientes reales de aprendizaje, ofrece al estudiante la posibilidad de implementar el proceso de atención de enfermería (PAE), método para brindar el cuidado enfermero, bajo la asesoría y guía del docente, contribuyendo así a su formación integral. (Meléndez, 2020)

Representando la oportunidad de integrar y aplicar saberes teóricos, heurísticos y axiológicos así como desarrollar habilidades para resolución de problemas, en situaciones 
clínicas; proceso formativo que tiene lugar en ambientes sociales que para los estudiantes, resultan a menudo complejos y nuevos, en los que se ha identificado al docente clínico como uno de los actores principales cuya función primordial es orientar y guiar la actividad mental constructiva de los alumnos, proporcionando ayuda pedagógica acorde a sus competencias, propiciando la comprensión y actuación autónoma de los alumnos. (Uriarte, Ponce, \& Bernal, 2016)

Sin embargo la participación docente no es aislada, se reconoce que debe establecerse estrecha relación entre las figuras enfermera docente y enfermeras clínicas, por lo que, a fin de potenciar el desarrollo de los estudiantes, resulta de interés que el docente conozca el perfil de las enfermeras de los diferentes servicios e identifique el grado de compromiso que tienen para con la enseñanza tutelar. (Cervera, 2018)

Al respecto, Joaquín et.al, (2020) señalan que, los estudiantes identifican que las interrelaciones establecidas con enfermeras de servicio durante las prácticas clínicas son un factor para el desarrollo de su capacidad de autonomía en la práctica del cuidado enfermero, a través de adquirir habilidades por medio de entrenamiento brindado y supervisado.

Gray \& Smith (2000, citado por Cook \& Álvarez, 2009) hacen referencia a la opinión de los estudiantes sobre la importancia de tener un docente clínico, cuyo rol debe ser de asesor y supervisor durante la práctica clínica, señalando además que, debe poseer características personales como ser agradable, comprensivo y tener disposición para una buena comunicación.

Del mismo modo mencionan que, en la intención de otorgar un cuidado de calidad desean trabajar con docentes que tengan: experiencia, competencia clínicas y conocimientos en el área disciplinar y de su especialidad, a fin de que se desempeñen como guía clara para los estudiantes durante el desarrollo de la práctica clínica.

Los estudiantes consideran necesaria la incorporación del docente clínico en donde se realiza la práctica clínica, toda vez que, al ser identificado en los diferentes servicios, podría asegurar su ayuda. (Cook \& Álvarez, 2009). Al respecto, Lorente Gallego et al. (2009, citado por Uriarte S. et.al) refieren que la alta satisfacción manifestada por alumnos en relación a sus prácticas en centros sanitarios se debe a la integración del profesional-tutor en la formación de pregrado, participando en las actividades prácticas e integración del alumno en el equipo de trabajo. 
Otros autores refieren que los alumnos consideran como aspecto sobresaliente que influye en el desarrollo de conocimientos, la utilización de estrategias de enseñanza y de aprendizaje, tales como la demostración y el ensayo para el aprendizaje o desarrollo de habilidades clínicas, así como la discusión académica de los objetivos de aprendizaje. Por su parte, los docentes, describen su rol como diverso, complejo y multifacético, realizando actividades orientadas a la organización de los diversos factores o elementos del campo clínico lo que es fundamental para que los estudiantes transiten exitosamente de un área clínica a otra (Lambert \& Glacken, 2006, citado por Cook \& Álvarez, 2009). Asimismo conceden particular importancia a la asignación de un tutor profesional calificado, cuyo papel será apoyar y evaluar al estudiante durante la práctica clínica y que conjuntamente con el docente, facilitarán el aprendizaje con actitud positiva y haciendo uso de estrategias varias (Gray \& Smith, 2000; Hanson \& Stenvig, 2008; Tsai \& Tsai, 2004, citado por Cook \& Alvarez, 2009).

Es importante tener presente que el estudiante de enfermería requiere bases teóricas solidas que aplicará en la práctica, requiriendo ser guiado por docentes o pares que le orientan durante este proceso, ya sea positiva o negativamente. Los profesionales clínicos deben estar en constante actualización de conocimientos teóricos e integrarlos en su práctica diaria, considerando que fungen como modelos a seguir por futuras generaciones. (Molina Chailán, Pía Mabel, \& Jara Concha, Patricia del Tránsito, 2010).

No debe obviarse la carga emocional que conlleva la realización de prácticas clínicas, Sevilla (2014, citado por Tessa R., 2014) señala que al iniciar, existe temor, ansiedad y dificultades, derivado principalmente por la falta de experiencia, situación que es esperado disminuya a medida que van adquiriendo confianza en sí mismos y empiezan a asumir el rol profesional futuro.

Relacionado con ello, Pulido et. al (2016) refieren que al incursionar en la práctica, los estudiantes tienen un acercamiento real a su futura profesión y con frecuencia se enfrentan a situaciones difíciles que les generan estrés. Destacando la necesidad de formarles en habilidades emocionales que les favorezcan afrontar de manera más adecuada y positiva sus prácticas clínicas, redundando en un mejor entrenamiento como futuros profesionales de enfermería. Asimismo proponen que esa formación se haga extensiva a las enfermeras asistenciales mismas que tiene un rol importante en la formación de alumnos, ya que al 
desempeñarse como tutoras, su experiencia y comportamiento ante situaciones complejas y difíciles sirven de modelaje.

Se ha identificado que la no congruencia entre contenidos teóricos revisados previamente y lo que observan o vivencian durante las prácticas, genera en el estudiante temor y angustia como resultado de no poder relacionarlos y en consecuencia no poder aplicarlos o ejecutarlos, lo que constituye obstáculos en el aprendizaje. Siendo importante recalcar que el conocimiento de esta situación, es un elemento valioso para plantear metodologías de trabajo que logren disminuir dichas dificultades. Tessa (2014)

Así, la planeación y desarrollo de la enseñanza clínica incluye: Determinar áreas o servicios clínicos en función de los objetivos de aprendizaje o competencias a desarrollar, selección de estrategias de enseñanza y de aprendizaje, creación de un ambiente de aprendizaje estimulante, establecer métodos y medios de supervisión así como diversas formas de evaluación atendiendo a las características del estudiante y su contexto. Al respecto, Uriarte (2016) refiere que "una mejor comprensión de la calidad de educación de la práctica clínica desde la perspectiva de los estudiantes será muy útil en la prestación de mejores experiencias educativas para éstos”. Para ello, el conjunto de elementos que se conjugan en la práctica clínica, requieren ser analizados y evaluados,

Sin discusión, la evaluación forma parte de todo proceso educativo, es muy amplia y su aplicación es variada dependiendo de contexto, criterios y fines, respecto a sus características, para este trabajo, resulta importante las mencionadas por Sanmartí (2014, citado por Espinoza, 2017) y que son las siguientes: Obtención de información, análisis de la misma y toma de decisiones pedagógicas, formativas y reguladoras, identificando áreas de oportunidad para apoyar el proceso formativo disciplinar y social de los estudiantes.

Características a considerar cuando se visualiza la necesidad de enfrentar el reto de formación de profesionales de ciencias de la salud competentes para desempeñarse en el contexto internacional. A este respecto Espinoza (2017) refiere que las metodologías de evaluación orientadas a asegurar la calidad de los procesos educativos resultan relevantes en investigaciones donde la evaluación es compartida entre docentes universitarios y profesionales de la salud que evalúan a los estudiantes en ámbitos clínicos. De acuerdo a Brailovsky, (2008, citado por Espinoza, 2017) en materia de evaluación clínica se espera 
que cada estudiante sea evaluado en varias ocasiones por distintos docentes y en diversas situaciones clínicas

Específicamente en el área de enfermería, se hace evidente con el fin de integrar aspectos teóricos en escenarios clínicos, el uso de estrategias metodológicas diversas , que evalúan habilidades y actitudes además de conocimientos, es decir, "lo que las personas deben saber, saber hacer y hacer (competencias)" . (De la Horra,2010, citado por Espinoza 2017) Siendo necesaria una evaluación completa de los espacios o campos clínicos de enseñanza, acción que es de vital importancia en funcion de retroalimentar aspectos tales como: Formación de los alumnos, atención que brindan a los usuarios, participación del personal de enfermería, desempeño docente y oportunidades de aprendizaje determinadas por infraestructura, equipamiento de la unidad médica, tipo de usuarios e interrelaciones personales.

Esta evaluación se constituye en un complemento valioso de información para las instituciones educativas formadoras de recurso humanos de enfermería, mismas que en respuesta a lineamientos nacionales elaborados y propuestos por el Comité de Evaluación de la CIFRHS, para obtener Reconocimiento de Validez Oficial (RVOE) por la SEP, deben cumplir con ciertos criterios que, en materia de Campos clínicos y organización académica administrativa, hacen referencia, entre otros, a: Planeación de docencia clínica (organización entre asignatura, unidad de aprendizaje o módulo, profesor, campo clínico y académico - administrativa), campos clínicos solicitados congruentes con perfil profesional, matrícula proyectada, número de alumnos asignados por docente $\mathrm{y}$ distribución de los mismos de acuerdo a indicadores de las instituciones de salud.(Gobierno de México, 2019)

En la misma idea, pero con fines de acreditación o reacreditación, existe también alineación a lo establecido en indicadores de la categoría de Plan de estudios orientados a Unidades de Aprendizaje de la disciplina que incluyen, entre otros, el plan de práctica clínica por cada unidad de aprendizaje teórico-práctico. (Consejo Mexicano para la Acreditación de Enfermería, 2019)

Atendiendo a todo lo anteriormente mencionado y considerando la participación como docentes de enfermería, es que surge el interés por realizar la presente investigación, planteando los objetivos que se señalan a continuación.

\section{OBJETIVOS}




\section{General}

- Evaluar un campo cínico de enfermería del área materno infantil.

\section{Específicos}

\section{Valorar las dimensiones:}

- Organización a través de evaluar: Condiciones o características de campo clínico, horario y duración de la clínica

- Ambiente laboral y de aprendizaje, por medio de evaluar: Entorno, condiciones, experiencia vivida, relaciones con los profesionales como persona y como alumno en formación

- Relación teoría-práctica, evaluando: Relación con experiencias educativas precedentes, capacitación para desempeño en el área de pediatría y

- Seguimiento y evaluación a través de evaluar: Sistema de evaluación, seguimiento o supervisión por personal operativo y docente, cumplimiento con productos de aprendizaje

\section{METODOLOGÍA}

\section{Tipo de estudio y diseño metodológico}

Estudio cuantitativo, descriptivo, longitudinal, con cortes anuales, efectuado de 2015 a 2019 en un Hospital Escuela, de 2do nivel de la ciudad de Xalapa, Veracruz, que es utilizado como campo clínico del área materno infantil.

\section{Técnica de investigación e instrumentos}

La técnica utilizada fue la encuesta y como instrumento se utilizó un cuestionario, diseñado exprofeso y validado con alfa de Cronbach, que explora, a través de 12 ítems con opciones de respuesta dicotómicas, cuatro dimensiones:

1) Organización (condiciones o características de campo clínico, horario y duración de la clínica)

2) Ambiente laboral y de aprendizaje (Entorno, condiciones, experiencia vivida, relaciones con los profesionales como persona y como alumno en formación),

3) Relación teoría-práctica (relación con experiencias educativas precedentes, capacitación para desempeño en el área de pediatría) y

4) Seguimiento y evaluación (Sistema de evaluación, seguimiento o supervisión por personal operativo y docente, cumplimiento con productos de aprendizaje)

Dicho cuestionario fue aplicado a los estudiantes al concluir el periodo de práctica.

\section{Población y muestra}


La población estuvo constituida por estudiantes de licenciatura en enfermería de una universidad pública que cursaron, durante el periodo de estudio, la experiencia educativa clínica.

La muestra estuvo constituida por el total de alumnos que tuvieron como campo clínico el hospital seleccionado, siendo un total de 81, atendiendo a los criterios de selección establecidos.

\section{Criterios de selección}

\section{Inclusión}

\section{Alumnos que:}

1) Cursaron la experiencia educativa en el campo clínica seleccionado en los periodos febrero-julio y agosto - enero de los años 2015 al 2019, en el turno vespertino.

2) Que aceptaron participar y firmaron consentimiento informado

\section{Exclusión}

\section{Alumnos que:}

1) Cursaron la experiencia educativa en dichos periodos y mismo campo clínico, en el turno matutino.

2) Cursaron la experiencia educativa en mismos periodos, campo clínico y turno, procedentes de otra institución educativa.

3) Que no aceptaron participar.

\section{Eliminación}

1) Cuestionarios contestados de manera incompleta.

2) Alumnos que desistieran de participar

\section{RESULTADOS}

\section{Dimensión organización.}

Referente a si las condiciones o características del campo clínico y horario son adecuados (ítems 1 y 2), los porcentajes de respuestas afirmativas son similares, con ligeras variaciones en los primeros tres años alcanzando el 100\% en el 2019; llamando la atención que respecto a si la duración de la clínica es adecuada (ítem 3), el número de respuestas negativas se incrementó alcanzando en el último año valor de $25 \%$, siendo estadísticamente significativo. (Gráfica 1) 


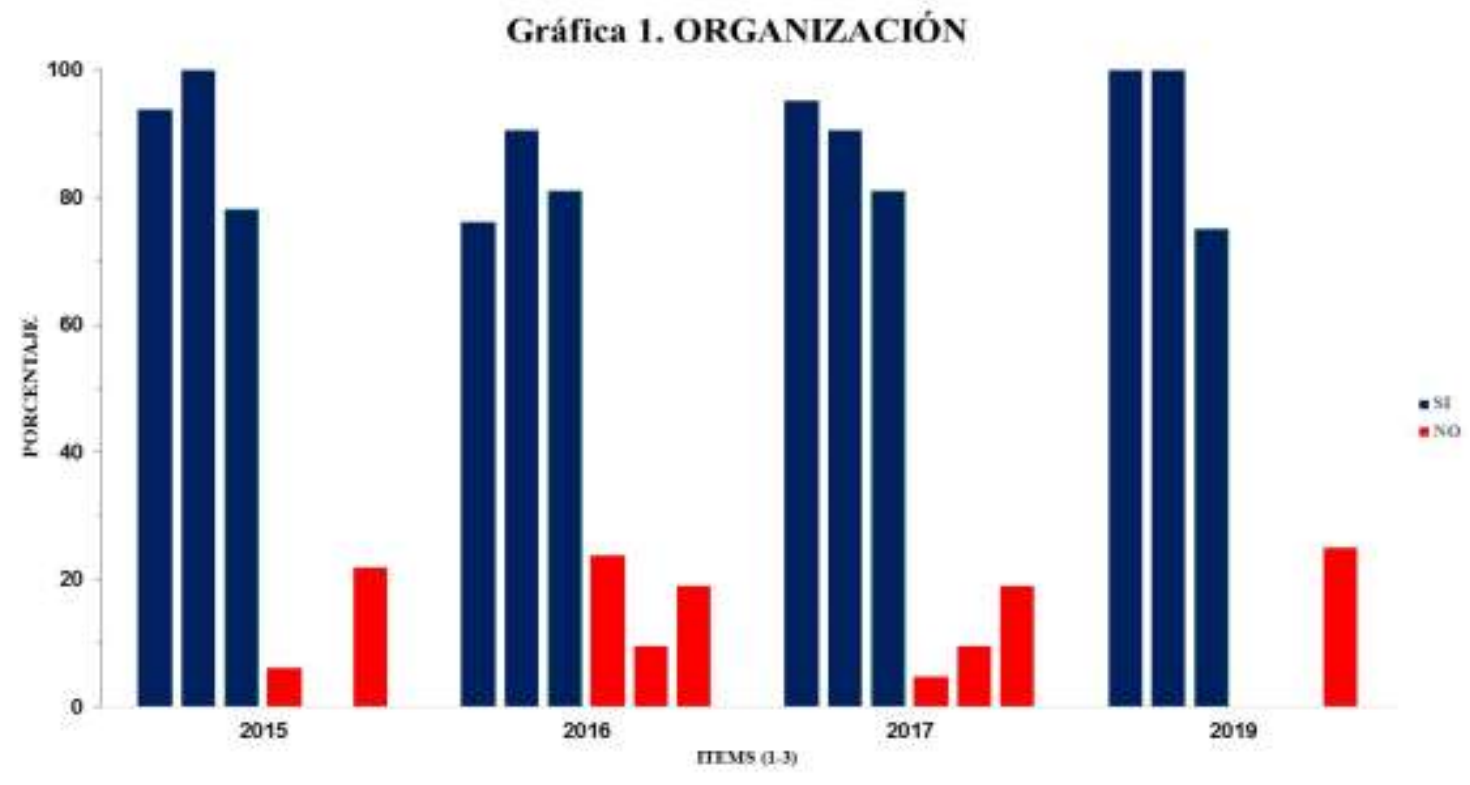

Dimensión ambiente laboral y de aprendizaje

Respecto a si el entorno, las condiciones y la experiencia vivida en la clínica fueron motivadores (ítem 4) se observó un incremento gradual llegando al 100\% en el último año. Para el aspecto de si las relaciones mantenidas con los profesionales (personal operativo) del campo clínico les fueron satisfactorias como persona y como estudiante en formación (ítems 5 y 6) las respuestas afirmativas registran variaciones que van de $96 \%$ y $100 \%$ a $94 \%$. (Gráfica 2)

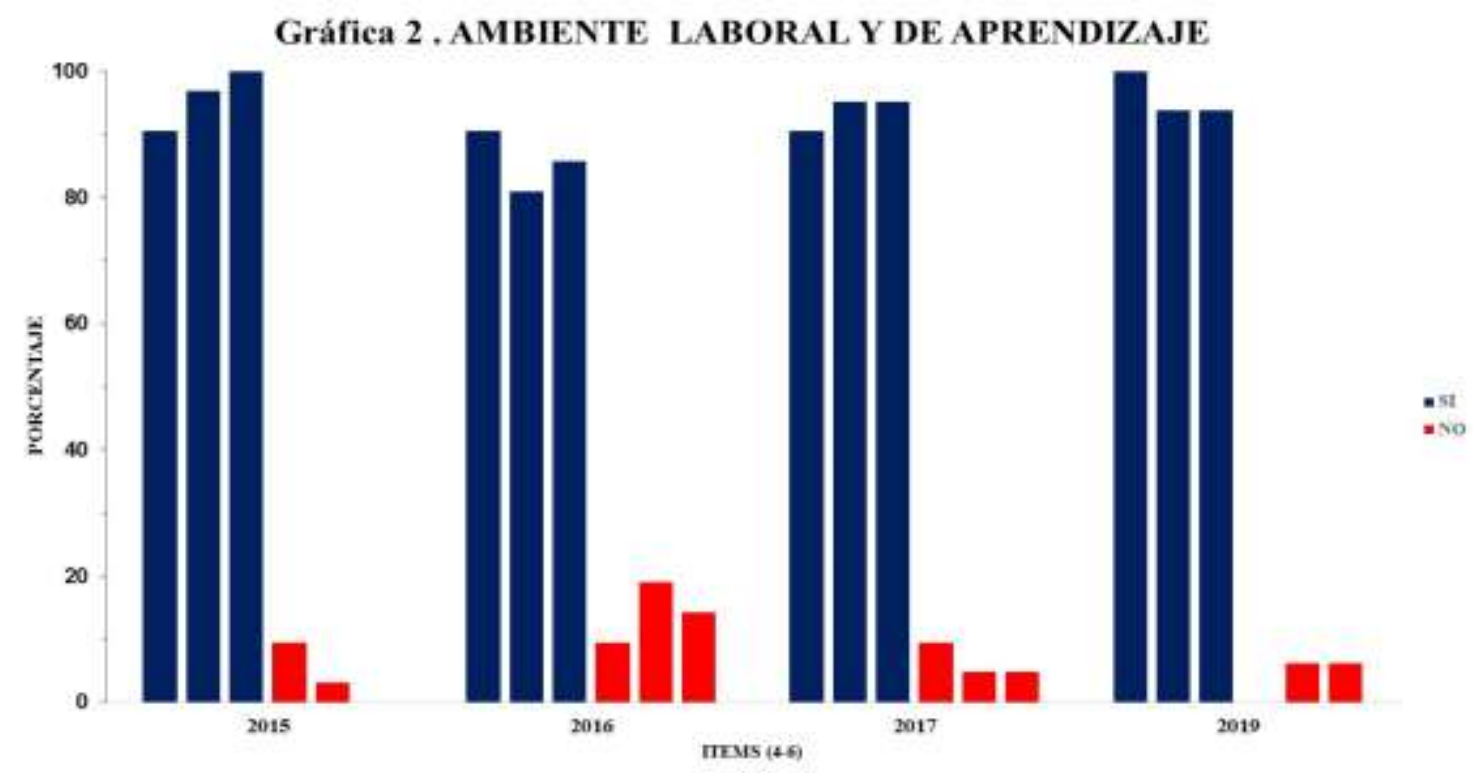

\section{Dimensión relación teoría-práctica}


En cuanto a si existe correlación entre saberes adquiridos en experiencias educativas cursadas previamente y los que requirieron y pusieron en práctica durante el desarrollo de la clínica (ítem 7), el porcentaje de respuestas afirmativas se mantuvo por arriba del 80\%. (Gráfica 3)

En porcentaje menor a 80\% (años 2016 y 2019), los estudiantes consideran que la clínica les ha capacitado para desempeñarse en servicios del área materno infantil (ítem 8). (Gráfica 3) Resultados esperados si se toma en cuenta que refirieron que la duración de la práctica debe ser mayor.

\section{Gráfica 3. RELACIÓN TEORÍA-PRÁCTICA}

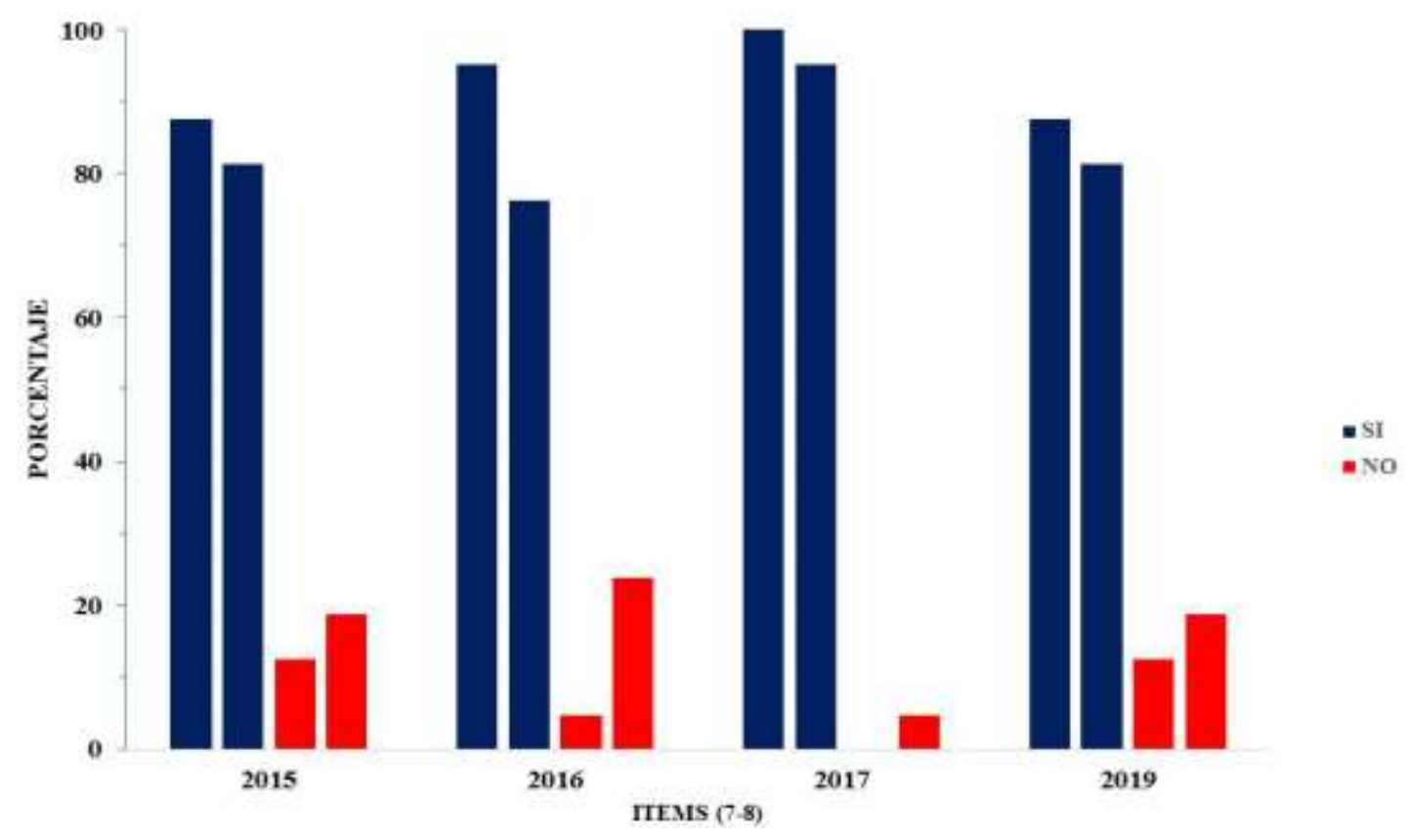

\section{Seguimiento y evaluación}

Se observa porcentaje mayor de $80 \%$ para quienes consideran que el sistema de evaluación, así como el seguimiento y supervisión por parte del personal operativo y docente son adecuados (ítems 9,10 y 11). Llamando la atención que se registra decremento progresivo en el cumplimiento de entrega de productos de aprendizaje (ítem 12), alcanzando $63 \%$ de incumplimiento en el último año, siendo estadísticamente significativo. 


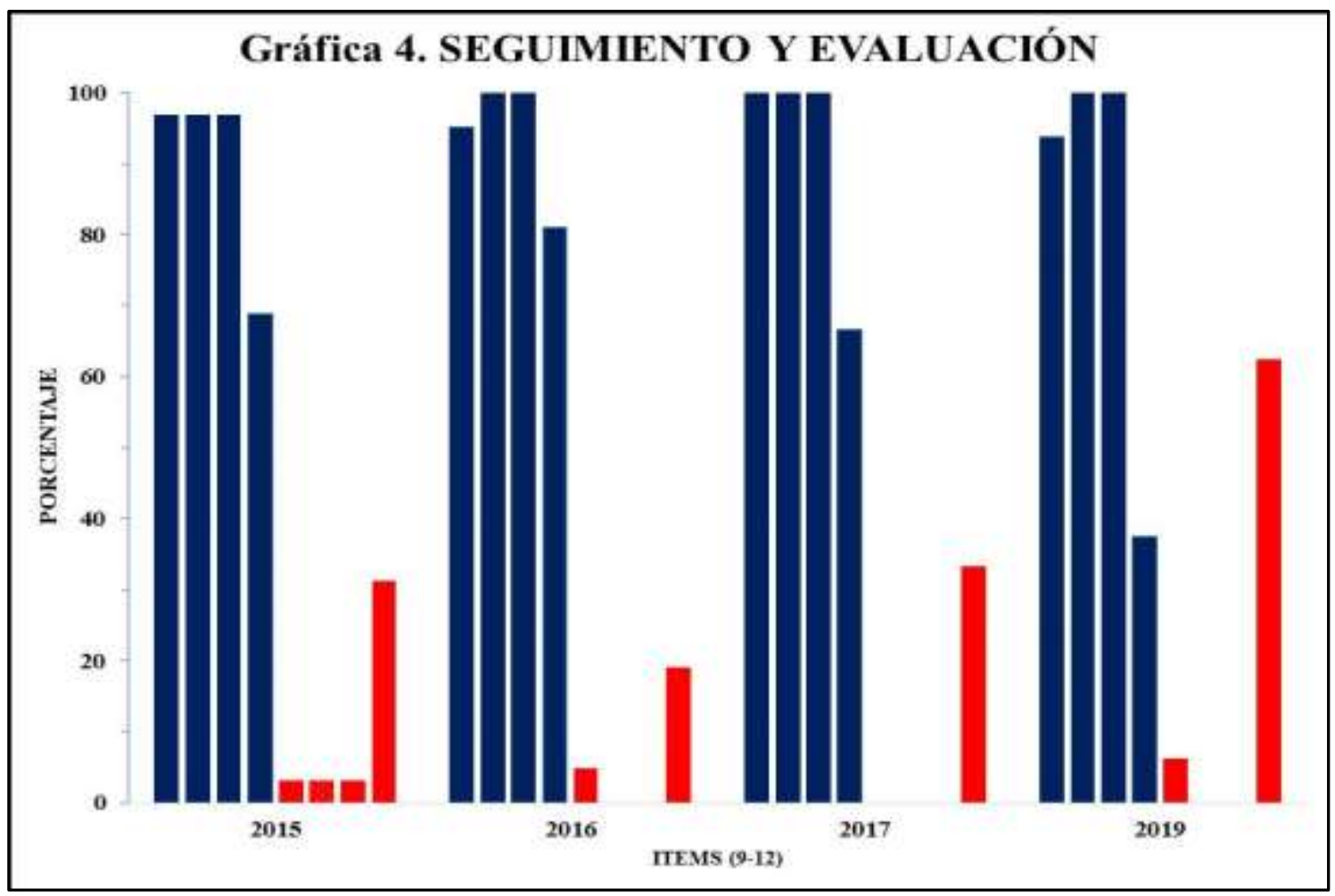

\section{CONCLUSIÓN}

En relación al objetivo general, los resultados obtenidos reflejan que el campo clínico es adecuado lo que se puede traducir en una buena oportunidad para el desarrollo de la experiencia educativa clínica.

Referente a objetivos específicos, las dimensiones organización y ambiente laboral y de aprendizaje, fueron evaluadas positivamente, al considerar en porcentaje superior a $90 \%$, que el entorno y la experiencia vivida fueron motivadoras, misma situación en lo que respecta a relaciones interpersonales con el personal operativo señaladas como satisfactorias en la dualidad persona/ estudiante en formación.

Las dimensiones relación teoría-práctica y seguimiento y evaluación, fueron calificadas positivamente en porcentaje mayor a $80 \%$, al referir correspondencia entre saberes previos y los requeridos y puestos en práctica durante el desarrollo de la clínica, asimismo el seguimiento, evaluación y supervisión por parte del personal operativo y docente fueron catalogados como adecuados.

Sin embargo, quedan de manifiesto áreas de oportunidad que requieren atención al interior de la entidad educativa, tales como: Duración de la práctica y su efecto en el desempeño en atención materno infantil, correlación entre saberes adquiridos previamente y los requeridos para la práctica y cumplimiento con productos de 
aprendizaje. Aspectos que denotan, probablemente mayor beneficio si permanecieran más tiempo en el campo clínico y existiera mayor correlación entre saberes previos y los requeridos para la práctica. Resultando relevante el incumplimiento en la entrega de productos de aprendizaje, lo que lleva a que el docente revise los criterios establecidos para evidencias de aprendizaje. Por lo que se recomiendan como puntos de buena praxis, que se busquen estrategias de diseño metodológico para atender los puntos antes señalados y que el estudio se amplíe a una muestra probabilística, a fin de que la comunidad académica identifique puntos sobre los cuales incidir, mediante análisis, reflexión, discusión académica e investigación educativa, en la intención de mejorar la enseñanza clínica.

\section{REFERENCIAS BIBLIOGRÁFICAS}

Brailovsky C. 2008, citado por Espinoza F. B. (2017). La evaluación de competencias clínicas en estudiantes de enfermería, un nuevo paradigma. Validación de rúbrica. Tesis doctoral. Obtenido de https://www.tesisenred.net/bitstream/handle/10803/587111/2018_Tesis_Espinoz $\underline{\text { a } \% 20 F e r n a n d e z}$ Maria\%20Bruna.pdf?sequence $=1 \&$ isAllowed $=y$

Cervera-Gasch A., Gonzalez-Chorda V., Mena-Tudela D., Salas-Medina P., Folch-Ayora A, Macia-Soler L.(2017). Participación de las enfermeras clínicas en la formación práctica de estudiantes de Grado en Enfermería. Obtenido de https://www.elsevier.es/es-revista-enfermeria-clinica-35-linkresolverparticipacion-las-enfermeras-clinicas-formacion-S1130862117301912

Consejo Mexicano para la Acreditación de Enfermería AC. 2019. Instrumento de evaluación para la acreditación de programas académicos de licenciatura en enfermería SNAE-2019. Obtenido de: http://www.comace.mx/Documentos/INSTRUMENTO_SNAE-2019.pdf

Cook, M. P., \& Alvarez, E. (2009). Características del docente clínico de enfermería. Horizonte de enfermería 20 (2), 53-61. Obtenido de http://horizonteenfermeria.uc.cl/images/pdf/20\%202/caracteristicas_docentes.pdf

De la Horra Gutierrez I, Beneit Montesinos J.2010), citado por Espinoza F. B.2017. La evaluación de competencias clínicas en estudiantes de enfermería, un nuevo paradigma. Validación de rúbrica. Tesis doctoral. Obtenido de 
https://www.tesisenred.net/bitstream/handle/10803/587111/2018_Tesis_Espinoz $\underline{\text { a } \% 20 \text { Fernandez_Maria\%20Bruna.pdf?sequence }=1 \& \text { isAllowed }=y}$

Durante, E.2012, citado por Maroto 2017. Evaluación de los aprendizajes en escenarios clínicos: ¿Qué evaluar y por qué? Revista Educación 41(1), 1-18, e-ISSN: 22152644, enero-junio, 2017. Obtenido de: https://www.scielo.sa.cr/pdf/edu/v41n1/2215-2644-edu-41-01-00133.pdf

Espinoza F. B. (2017). La evaluación de competencias clínicas en estudiantes de enfermería, un nuevo paradigma. Validación de rúbrica. Tesis doctoral. Obtenido de

https://www.tesisenred.net/bitstream/handle/10803/587111/2018_Tesis_Espinoz a\%20Fernandez_Maria\%20Bruna.pdf?sequence=1\&isAllowed=y

Gray \& Smith (2000), citado por Cook \& Álvarez ,E. (2009) Características del docente clínico de enfermería. Horizonte de enfermería 20 (2), 53-61. Obtenido de http://horizonteenfermeria.uc.cl/images/pdf/20\%202/caracteristicas_docentes.pdf

Gobierno de México. Secretaría de Salud. Dirección de calidad y educación. 2019. Criterios esenciales para evaluar planes y programas de estudio de las licenciaturas en enfermería. Obtenido de: http://www.cifrhs.salud.gob.mx/site1/planesprogramas/docs/200325_ce_enfermeria_v4.pdf

Joaquin A.D.L., Cuevas G. L., López S. M.G., Bravo S.L., Valadez D. D. (2020). Experiencias en campos clínicos de estudiantes de diferentes escuelas de Enfermería. Revista Ocronos. Vol. III. No 1 - Mayo 2020. Obtenido de https://revistamedica.com/experiencias-campos-clinicos-estudiantes-enfermeria/ Lambert, V., \& Glacken, M. (2006) citado por Cook, M. P., \& Álvarez, E. (2009). Características del docente clínico de enfermería. Horizonte de enfermería 20 (2), 53-61. Obtenido de http://horizonteenfermeria.uc.cl/images/pdf/20\%202/caracteristicas_docentes.pdf

Lorente-Gallego AM, García Sánchez C, López Arroyo MJ, et al. (2009) citado por Uriarte, S., Ponce, G., \& Bernal, M. (2016). Vivencias cotidianas en espacios clínicos. Enfermería Universitaria 13 (3), 171-177. 
Maroto-Marín. O. 2017. Evaluación de los aprendizajes en escenarios clínicos: ¿Qué evaluar y por qué? Revista Educación 41(1), 1-18, e-ISSN: 2215-2644, enerojunio, 2017. Obtenido de: https://www.scielo.sa.cr/pdf/edu/v41n1/2215-2644edu-41-01-00133.pdf

Meléndez Chávez, Sendy. (2020). La importancia de la práctica en la formación de enfermería en tiempos de Covid-19: experiencias de alumnos. Revista Dilemas contemporáneos: educación, política y valores, 8(spe5), 00004. Epub 28 de enero de 2021.https://doi.org/10.46377/dilemas.v8i.2479

Molina Chailán, Pía Mabel, \& Jara Concha, Patricia del Tránsito. (2010). El saber práctico en Enfermería. Revista Cubana de Enfermería, 26(2) Obtenido de http://scielo.sld.cu/scielo.php?script=sci_arttext\&pid=S0864$\% 2003192010000200005 \& \operatorname{lng}=\mathrm{es} \& \operatorname{tn} \mathrm{ln}=\mathrm{es}$

Pulido-Martos, Manuel, Augusto-Landa, José M., \& López-Zafra, Esther. 2016. Estudiantes de Enfermería en prácticas clínicas: el rol de la inteligencia emocional en los estresores ocupacionales y bienestar psicológico. Index de Enfermería, 25(3), 215-219. Disponible en http://scielo.isciii.es/scielo.php?script=sci_arttext\&pid=S1132$12962016000200020 \& \operatorname{lng}=\mathrm{es} \& \ln \mathrm{l}=\mathrm{es}$

Sanmartí N. 2014, citado por Espinoza F. B. (2017). La evaluación de competencias clínicas en estudiantes de enfermería, un nuevo paradigma. Validación de rúbrica. Tesis doctoral. Obtenido de https://www.tesisenred.net/bitstream/handle/10803/587111/2018_Tesis_Espinoz a\%20Fernandez_Maria\%20Bruna.pdf?sequence $=1 \&$ isAllowed $=y$

Sevilla Casado M.2013, citado por Tessa R.2014. Dificultades percibidas por los estudiantes de Enfermería en su inserción a la práctica clínica de Enfermería Medicoquirúrgica. Rev. iberoam. Educ. investi. Enferm. 2014; 4(4):35-43. Obtenido de https://www.enfermeria21.com/revistas/aladefe/articulo/137/dificultadespercibidas-por-los-estudiantes-de-enfermeria-en-su-insercion-a-la-practicaclinica-de-enfermeria-medicoquirurgica/

Secretaría de Salud (2012). PROYECTO de Norma Oficial Mexicana PROY-NOM-010SSA3-2012, Educación en salud. Utilización de campos clínicos para las prácticas 
clínicas y el servicio social de enfermería. Obtenido de http://www.diariooficial.gvamundial.com.mx/historico/2012/Agosto/ss08_1.pdf

Tessa R.2014. Dificultades percibidas por los estudiantes de Enfermería en su inserción a la práctica clínica de Enfermería Medicoquirúrgica. Rev. iberoam. Educ. investi. Enferm. 2014; 4(4):35-43. Obtenido de https://www.enfermeria21.com/revistas/aladefe/articulo/137/dificultadespercibidas-por-los-estudiantes-de-enfermeria-en-su-insercion-a-la-practicaclinica-de-enfermeria-medicoquirurgica/

Uriarte, S., Ponce, G., \& Bernal, M. (2016). Vivencias cotidianas en espacios clínicos. Enfermería Universitaria 13 (3), 171-177. Obtenido de http://revistaenfermeria.unam.mx/ojs/index.php/enfermeriauniversitaria/article/view/80 